\title{
Microdynamical Effects on Momentum Distribution in Stellar Plasmas
}

\author{
Fatima Bachari* \\ Politecnico di Torino - Dipartimento di Fisica, Italy \\ E-mail: fatima.bachariapolito.it
}

\section{Fabrizio Ferro}

Politecnico di Torino - Dipartimento di Fisica and INFN - Sezione di Torino, Italy

E-mail: fabrizio.ferro@polito.it

\section{Giancarlo Maero}

Gesellschaft für Schwerionenforschung (GSI) - Darmstadt, Germany

E-mail: g.maero@gsi.de

\section{Piero Quarati}

Politecnico Torino - Dipartimento di Fisica and INFN - Sezione di Cagliari, Italy

E-mail: piero.quarati@polito.it

\begin{abstract}
We show that, if a random force is present, the microscopic dynamics of ion elastic collisions and quantum effects may sensibly modify the momentum distribution of ions and electrons in stellar plasmas. We also show that a few microscopic interactions among the particles, that are significant in very specific energy intervals, lead to peculiar slight corrections to the usual MaxwellBoltzmann distribution. All these modifications can be easily taken into account by using the nonextensive statistical mechanics. Consequences on the resonant and non-resonant fusion rates are remarkable and may affect strongly some astrophysical processes. A few examples are reported.
\end{abstract}

PACS: $52.72 .+v, 05.20 .-y, 51.10 .+y, 95.30 . D r$

International Symposium on Nuclear Astrophysics - Nuclei in the Cosmos - IX

25-30 June 2006

CERN

${ }^{*}$ Speaker. 


\section{Introduction}

In a dense neutral coupled stellar plasma (e.g. in white dwarf cores), a random force field $\mathscr{F}$ may be present owing to either random microfields induced by thermal fluctuations on the ion positions [1, 2], or dissipative random forces [3].

In the presence of the field $\mathscr{F}$, different types of interactions among the ions modify the tail of the ion momentum distribution in characteristic energy ranges with respect to a pure MaxwellBoltzmann distribution (MB) [4]. These corrections to the MB distribution may be interpreted within the framework of the nonextensive statistical mechanics [5] where, as we shall show, the entropic parameter can be expressed in terms of the collision cross sections of the various nonnegligible interactions. In particular, we discuss the contributions [6] of the ion-dipole and the screened ion-ion interactions, and the quantum effect owing to many-body collisions: the latter leads to the appearance of power-law tail in the momentum distribution.

We shall then sketch a possible link between the microscopical dynamics of ions and the nonextensive statistical mechanics, which allows the interpretation of the deformed momentum distribution as an equilibrium distribution, although slightly differing from MB.

\section{Kinetic equation under a generalized random force}

Adapting the model discussed in [7], aiming at a description of a plasma in presence of a generic force field $\mathscr{F}$, the relevant kinetic equation for the isotropic part of the 1-body ion momentum distribution function $f=f(v)$ reads

$$
\pm \frac{2}{3} \frac{\mathscr{F}^{2}}{\mu^{2} v^{2}} \frac{\mathrm{d} f}{\mathrm{~d} v}+\kappa\left(v f+\frac{k_{\mathrm{B}} T}{\mu} \frac{\mathrm{d} f}{\mathrm{~d} v}\right)=0,
$$

where $v(v)$ is the collision frequency, $\kappa=2 \mu^{2} / m^{2}$ the energy transfer coefficient and the \pm sign refers to the two cases of sub- or super-diffusivity (e.g. microfields enhance the diffusivity), respectively.

The analytical solution of Eq. 2.1 may be written as

$$
f(v) \propto \exp \left[-\int_{0}^{v} \mathrm{~d} v^{\prime} \frac{\mu v^{\prime}}{k_{\mathrm{B}} T \pm \frac{2}{3} \frac{\mathscr{F}^{2}}{\mu \kappa v^{2}}}\right] .
$$

Two physical situations can be addressed here:

- $\mathscr{F}^{2} \ll v^{2} \kappa \mu k_{\mathrm{B}} T$ : Eq. 2.2 reduces to the MB distribution regardless of any assumption about the dependence of the collision frequency $v$ against the velocity;

- $\mathscr{F}^{2} \geq v^{2} \kappa \mu k_{\mathrm{B}} T: f(v)$ depends on the form of the collision frequency $v(v)=n v \sigma(v)$. The MB distribution can be recovered only if $\sigma_{0}(v)=\alpha_{0} v^{-1}$ ( $\alpha_{0}$ being a suitable constant), and the plasma temperature is renormalized as

$$
k_{\mathrm{B}} T_{\mathrm{eff}}=k_{\mathrm{B}} T \pm \frac{2}{3} \frac{\mathscr{F}^{2}}{\kappa \mu n^{2} \alpha_{0}^{2}},
$$


Since the limit of $f(v)$ in the absence of any field $\mathscr{F}$ must be the MB distribution, the previously defined $\sigma_{0} \propto v^{-1}$ cross section must always be present. Its origin is the interaction between an ion and an induced electric dipole [7].

In addition, we consider the effect of other two cross sections of interest in stellar plasmas, namely $\sigma_{1}(v) \equiv \alpha_{1}$, acting in non-ideal and weakly coupled stellar plasmas owing to the screened Coulomb interaction [8], and $\sigma_{2}(v) \equiv \alpha_{2} v$, related to the quantum energy-momentum uncertainty as we shall discuss later. We further assume absence of interference among $\sigma_{0}, \sigma_{1}$ and $\sigma_{2}$ as they act in separate velocity intervals.

\section{The ion momentum distribution}

In the case $\frac{T_{\text {eff }}}{T} \leq \frac{v_{1}^{4}}{4 v_{0}^{2} v_{2}^{2}}=\frac{\alpha_{1}^{4}}{4 \alpha_{0}^{2} \alpha_{2}^{2}}$, Eq. 2.2 gives

$$
f(v) \propto \exp \left(-\frac{\mu v^{2}}{2 k_{\mathrm{B}} T}\right) \times\left(\frac{2 c_{2} v^{2}+c_{1}-2 \sqrt{|K| c_{2}}}{2 c_{2} v^{2}+c_{1}+2 \sqrt{|K| c_{2}}}\right)^{\frac{\mu \tau}{4 k_{\mathrm{B}} T \sqrt{|K| c_{2}}}},
$$

where we made the definitions $c_{1} \equiv\left(\frac{\alpha_{1}}{\alpha_{0}}\right)^{2}, c_{2} \equiv\left(\frac{\alpha_{2}}{\alpha_{0}}\right)^{2}, \tau \equiv \frac{T_{\text {eff }}}{T}-1$, and $K \equiv-\frac{c_{1}^{2}}{4 c_{2}}+\tau+1$.

In this limit, the cross section $\sigma_{1}$ dominates and $\mathscr{F}$ does not play any role in the region of interest of astrophysical plasmas, as the perturbation from the MB distribution vanishes as $v \rightarrow+\infty$.

If, on the contrary, $\frac{T_{\text {eff }}}{T}>\frac{\alpha_{1}^{4}}{4 \alpha_{0}^{2} \alpha_{2}^{2}}$, we obtain

$$
f\left(\varepsilon_{p}\right) \propto \exp \left[-\frac{\varepsilon_{p}}{k_{\mathrm{B}} T_{\mathrm{eff}}}\right] \exp \left[-\delta\left(\frac{\varepsilon_{p}}{k_{\mathrm{B}} T_{\mathrm{eff}}}\right)^{2}\right] \exp \left[-\gamma\left(\frac{\varepsilon_{p}}{k_{\mathrm{B}} T_{\mathrm{eff}}}\right)^{3}\right],
$$

where $\varepsilon_{p}=\frac{p^{2}}{2 \mu}$ is centre-of-mass kinetic energy,

$$
\delta= \pm \frac{2}{3} \frac{\mathscr{F}^{2}}{\kappa \mu^{2} n^{2}} \frac{\alpha_{1}^{2}}{\alpha_{0}^{4}}
$$

and

$$
\gamma= \pm \frac{8}{9} \frac{\mathscr{F}^{2} k_{\mathrm{B}} T}{\kappa \mu^{3} n^{2}} \frac{\alpha_{2}^{2}}{\alpha_{0}^{4}}\left(1-\frac{\alpha_{1}^{4}}{\alpha_{0}^{2} \alpha_{2}^{2}}\right)+\frac{16}{27} \frac{\mathscr{F}^{4}}{\kappa^{2} \mu^{4} n^{4}} \frac{\alpha_{2}^{2}}{\alpha_{0}^{6}}
$$

Eq. 3.1, with $\gamma=0$ was proposed in the past [9] as an ad hoc distribution function for solving the puzzling problem of the solar neutrinos. On the contrary, we derived it from a purely kinetic equation.

From Eqs. 3.2 and 3.3, it is evident that the cross section $\sigma_{1}$ produces a correction to the first order, whilst $\sigma_{2}$ contributes to the second order only. In both cases, the force field $\mathscr{F}$ is the source of the deformations upon the ion momentum distribution.

We distinguish three different energy intervals where the factors in Eq. 3.1 give the largest contribution. If $\varepsilon_{p} \sim k_{\mathrm{B}} T_{\mathrm{eff}}$, the MB factor dominates. If $\varepsilon_{p} \sim k_{\mathrm{B}} T_{\mathrm{eff}} /|\delta|$, the $\delta$-exponential factor (Druyvenstein factor) is of the same order of the MB factor while, if $\varepsilon_{p} \sim|\delta / \gamma| k_{\mathrm{B}} T_{\text {eff }}$, the $\gamma$-term is not negligible anymore. 


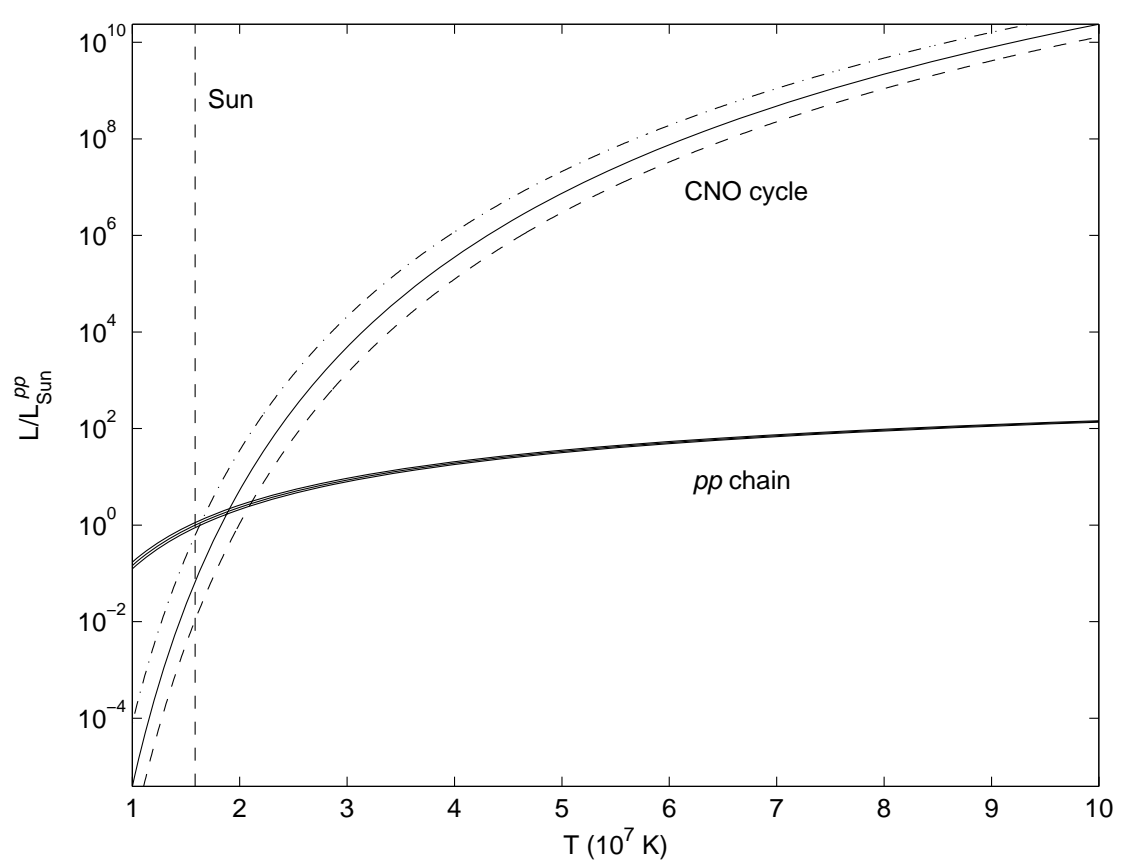

Figure 1: Log-linear plot of dimensionless luminosity over temperature, for the $p p$ chain and the CNO cycle, calculated for a plasma of density $\rho=100 \mathrm{~g} / \mathrm{cm}^{3}$ and a chemical composition $X_{H}=75 \%$ and $X_{N}=$ $0.8 \%$ for the hydrogen and nitrogen, respectively. (Dashed line, $\delta=+0.0045, q=0.991$; dash-dotted line, $\delta=-0.0045, q=1.009$ ). The vertical line shows the temperature of the Sun core. All curves are normalized with respect to the $p p$ luminosity. The $p p$ chain is slightly affected by nonextensivity (taken from [11]).

\section{Connection with the nonextensive statistical mechanics}

Within nonextensive statistical mechanics, the ion energy factor at equilibrium is

$$
f\left(\varepsilon_{p}\right) \propto\left[1-(1-q) \frac{\varepsilon_{p}}{k_{\mathrm{B}} T_{\mathrm{eff}}}\right]^{\frac{1}{1-q}},
$$

$q$ being the entropic parameter.

In the low deformation limit $(q-1) \frac{\varepsilon_{p}}{k_{\mathrm{B}} T_{\text {eff }}} \rightarrow 0$ (e.g. in stellar plasmas), Eq. 4.1 reduces to Eq. 3.1, provided $\delta=(1-q) / 2$. This condition then establishes a link between the entropic parameter $q$ and our $\delta$ of Eq. 3.2, related to the microfield strength and the ion collision cross sections, i.e. dynamical properties of the system. In the case of the electric microfields, it was shown [10] that $\delta \simeq 12 \Gamma^{2} \alpha^{4}$, where $\Gamma$ is the plasma parameter, and $0.4<\alpha<1$ [8].

Nonextensivity may have important effects in the study of stellar burning stages [11]. The plot in Fig. 1 shows that, in the case of the Sun itself, corrections arise for the CNO cycle, while the $p p$ chain is almost unaffected.

Since the CNO curves in Fig. 1 are strongly dominated by the ${ }^{14} N(p, \gamma){ }^{15} O$ reaction, being the slowest within the cycle, it is therefore possible to provide an analytical first order formula expressing the nonextensive $\mathrm{CNO}$ rate (proportional to the luminosity) as a function of the plasma 
temperature as follows [12],

$$
\begin{aligned}
r_{C N O} & =\frac{2^{5 / 2}}{3^{1 / 2}}\left(1+\frac{15}{4} \delta-\frac{7}{3} \delta \frac{E_{0}}{k_{\mathrm{B}} T}\right) N_{N} N_{H} \mu_{N H}^{-1 / 2} \times \\
& \times \frac{S\left(E_{0}\right)}{\left(k_{\mathrm{B}} T\right)^{1 / 2}}\left[1-\frac{4}{3} \delta \frac{E_{0}}{k_{\mathrm{B}} T}\left(\frac{S^{\prime}(0)}{S\left(E_{0}\right)} E_{0}+\frac{S^{\prime \prime}(0)}{S\left(E_{0}\right)} E_{0}^{2}\right)\right] \times \\
& \times\left(\frac{E_{0}}{k_{\mathrm{B}} T}\right)^{1 / 2} \exp \left(-\frac{3 E_{0}}{k_{\mathrm{B}} T}\right) \exp \left(-\Delta_{N H}\right),
\end{aligned}
$$

where $\mu_{N H}$ is the $H-N$ reduced mass, $N_{N}$ and $N_{H}$ are the nitrogen and hydrogen number densities, $E_{0}$ is the MB Gamow energy, $S, S^{\prime}$ and $S^{\prime \prime}$ are the astrophysical factor and its derivatives with respect to energy, and

$$
\Delta_{N H}\left(\delta, \tilde{E}_{0}\right)=-\frac{3 E_{0}}{k_{\mathrm{B}} T}\left[1-\left(1+\frac{5}{3} \delta \frac{\tilde{E}_{0}}{k_{\mathrm{B}} T}\right)\left(1+2 \delta \frac{\tilde{E}_{0}}{k_{\mathrm{B}} T}\right)^{-2 / 3}\right],
$$

where

$$
\tilde{E}_{0}=E_{0}\left(1-\frac{4}{3} \delta \frac{E_{0}}{k_{\mathrm{B}} T}\right)
$$

is the nonextensive Gamow energy.

Considering the Sun core, Eq. 4.2 simplifies to

$$
r_{C N O}\left[\mathrm{~cm}^{-3} \mathrm{~s}^{-1}\right] \sim 7.09 \cdot 10^{-42} \cdot N_{N}\left[\mathrm{~cm}^{-3}\right] \cdot N_{H}\left[\mathrm{~cm}^{-3}\right] \cdot(1-450.8 \delta) .
$$

\section{Low energy applications: the radiative recombination}

The calculations of the MB radiative recombination rate $\left(\alpha_{M B}\right)$ in electron cooling show a large discrepancy (up to a factor 5) with respect to the experimental data at low energies, as shown in Fig. 2.

Using the distribution 3.1 in the calculations, we obtained a modified rate $\alpha_{q}$ that may account for the observed enhancement [14]. The nonextensive distribution, in the study of this phenomenon, accounts for the subdiffusion between the electron and the ion bunches. The $\alpha_{M B} / \alpha_{q}$ ratio is plotted in Fig. 3 with respect to the temperature.

A last remark: both for the MB and the nonextensive case, as a first approximation we have used isotropic distributions; the study of anisotropic case, which presents a further slight complication in the nonextensive case, is under way.

\section{Quantum energy-momentum uncertainty in stellar plasmas}

Considering dense stellar plasmas, the ion energy $\varepsilon$ and momentum $\varepsilon_{p}$ should be considered as independent variables with a dispersion function $\mathscr{D}\left(\varepsilon, \varepsilon_{p}\right)$, owing to many-body effects and correlations $[15,16] . \mathscr{D}\left(\varepsilon, \varepsilon_{p}\right)$ is a Lorentz-like energy-momentum generalized distribution. The quantum energy-momentum indeterminacy leads to a power-like tail of the momentum distribution, as it may be shown that

$$
f\left(\varepsilon_{p}\right) \equiv \int \mathrm{d} \varepsilon \mathscr{D}\left(\varepsilon, \varepsilon_{p}\right)=\frac{2}{\pi^{3 / 2}} \frac{\sqrt{\varepsilon_{p}}}{\left(k_{\mathrm{B}} T\right)^{3 / 2}}\left[\exp \left(-\frac{\varepsilon_{p}}{k_{\mathrm{B}} T}\right)+\frac{2}{3 \pi} \text { const } \cdot\left(k_{\mathrm{B}} T\right)^{3 / 2} \frac{1}{\varepsilon_{p}^{4}}\right],
$$




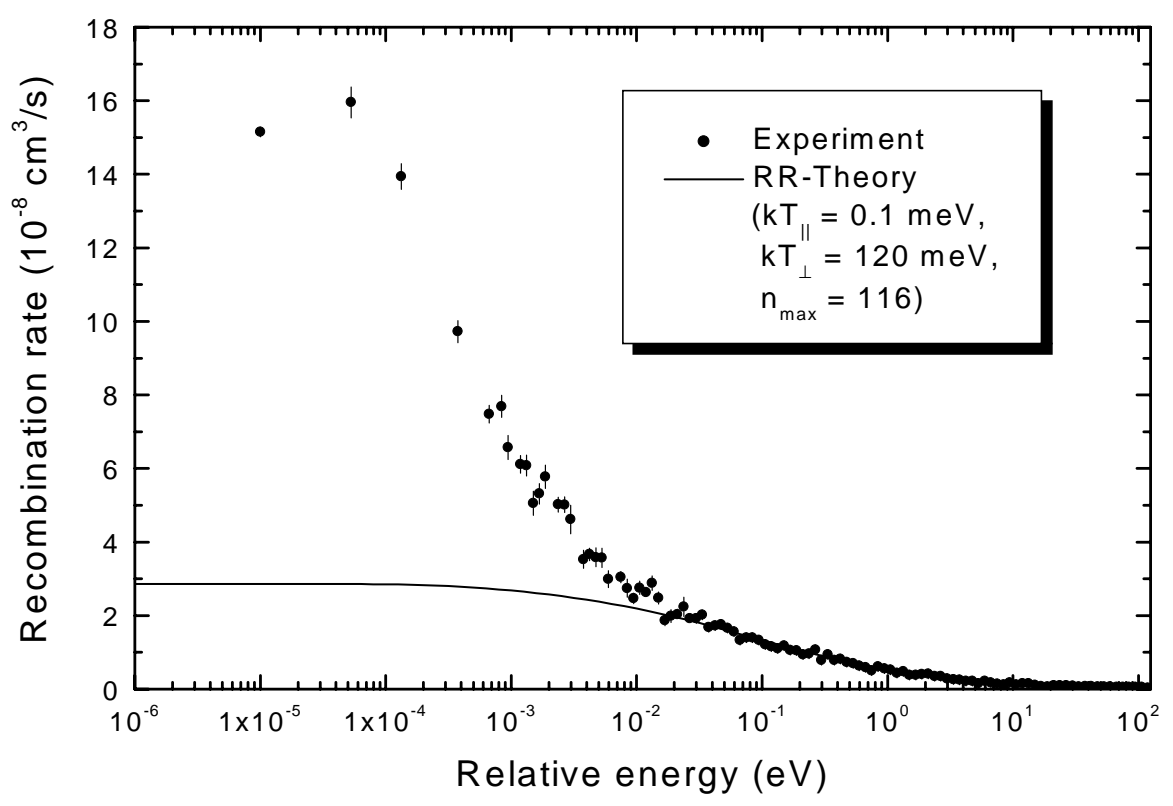

Figure 2: Comparison between $\mathrm{MB}$ calculations and experimental data in the case of the $\mathrm{Bi}^{83+}$ experiment (taken from [13]).

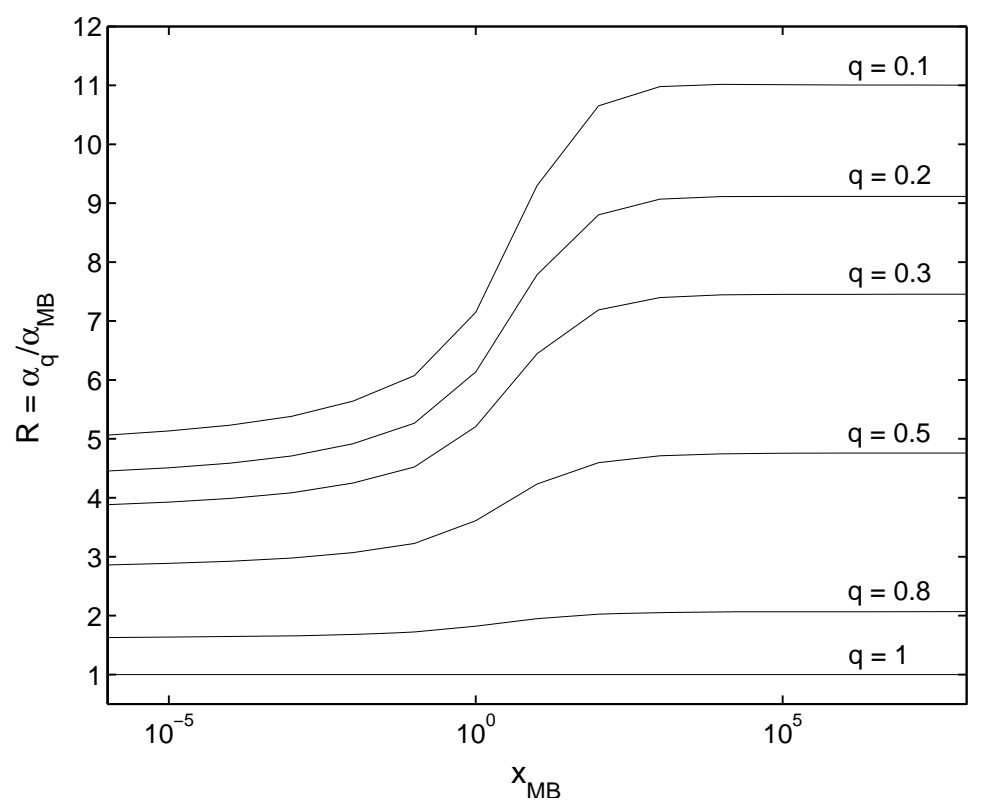

Figure 3: Plot of the recombination enhancement $R$ due to nonextensivity against the dimensionless temperature $x=k_{\mathrm{B}} T_{M B} / E_{\mathrm{Ryd}}$ for different values of the $q$ parameter in the superextensivity interval from $q=0.1$ to $q=1$ (with $\delta \equiv(1-q) / 2$ ). The calculations were performed for an electron-positron system. 
with important consequences in the calculations of nuclear fusion rates [11].

Moreover, the quantum momentum distribution 6.1 may be written as [17]

$$
f\left(\varepsilon_{p}\right)=\int_{0}^{+\infty} \mathrm{d} \varepsilon \exp \left(-\frac{\varepsilon}{k_{\mathrm{B}} T}\right) \cdot \delta_{\gamma}\left(\varepsilon, \varepsilon_{p}\right) .
$$

Quantum uncertainty and nonextensivity are two different and distinct causes of deformation of the MB distribution; nevertheless they lead to the same effect if the collision cross section is of a given type. In particular, if we impose that superextensivity and quantum uncertainty give the same modifications on the actual distribution function, we obtain that the underlying cross section must be [4]

$$
\sigma\left(\varepsilon_{p}\right)=\sigma_{2}\left(\varepsilon_{p}\right) \propto \sqrt{\varepsilon_{p}}, \sigma(v)=\sigma_{2}(v) \propto v .
$$

Therefore, the cross section $\sigma_{2}$ is the link between quantum-uncertainty and nonextensive statistical effects.

\section{The tidal-like force}

We discuss here a simple argument for showing from which type of interaction force the cross section $\sigma\left(\varepsilon_{p}\right) \sim \sqrt{\varepsilon_{p}}$ does come. A generic central force may be written as

$$
F(r)=f_{0}\left(\frac{r}{R_{0}}\right)^{-s}
$$

where $f_{0}$ is a constant, $R_{0}$ a characteristic distance of the two-body center of mass with respect to a given origin, and $s$ is either a negative or positive integer.

The collision cross section that can be derived from the interaction 7.1 reads [18]

$$
\sigma=\pi\left(f_{0} \frac{\mu}{|\mathbf{p}|^{2}}\right)^{2 /(s-1)} .
$$

For obtaining the $\sigma\left(\varepsilon_{p}\right) \propto \sqrt{\varepsilon_{p}}$ behaviour, we must set $s=-3$, such that

$$
F_{Q}(r)=\left\{\begin{array}{ll}
f_{Q_{0}}\left(\frac{r}{R_{0}}\right)^{3} & r \leq R_{0} \\
0 & r>R_{0}
\end{array} .\right.
$$

The force 7.2 has a tidal-like origin and acts globally over all the particles in the plasma. These conditions can be found if we assume that an attractive central force of intensity $f_{Q_{0}}$, centered at a distance $R_{0}$ from the center of mass of the two interacting particles is superimposed to the ion plasma.

Assuming an entropic parameter $q \sim 0.1$, and a proton (i.e $\mu c^{2} \approx 460 \mathrm{MeV}$ ) plasma of density $n \approx 10^{-14} \mathrm{fm}^{-3}$, with $R_{0} \approx 10^{5} \mathrm{fm}$, we obtain $f_{Q_{0}} \approx 10^{-12} \mathrm{MeV} / \mathrm{fm}$

The quantum uncertainty with collision cross section $\sigma\left(\varepsilon_{p}\right) \sim \sqrt{\varepsilon_{p}}$ gives a distribution function belonging to an equilibrium state, although different from the Maxwell-Boltzmann distribution. 


\section{Conclusions}

We showed that in physical conditions common in dense stellar plasmas, many non ideal effects arise, and the Maxwell-Boltzmann momentum distribution represents only a first-order approximation of the actual distribution. Corrections mainly occur in the high-energy regime, and they originate from the active types of collisions and, more precisely, from the microscopical dynamics of the system.

Furthermore, quantum corrections are present as well, and we have shown that the energymomentum uncertainty may be related to a $\sigma\left(\varepsilon_{p}\right) \propto \sqrt{\varepsilon_{p}}$ cross section.

All the previous correction may be interpreted within the general framework of the nonextensive statistical mechanics. Our momentum distribution, although differing from MB may be interpreted as a fully-equilibrium distribution.

\section{References}

[1] C. A. Iglesias, PRA 27 (1983) 2705.

[2] M. Yu. Romanovsky et al., Physica A 252 (1998) 488.

[3] L. E. Reichl, "Modern course in Statistical Physics", Wiley \& Sons, NY, 1998.

[4] F. Ferro and P. Quarati, Phys. Rev. E 71, (2005) 026408.

[5] C. Tsallis, http://tsallis.cat.cbpf.br/biblio.htm

[6] F. Ferro, A. Lavagno and P. Quarati, Eur. Phys. J. A 21 n. 3 (2004) 529.

[7] V. E. Golant et al., "Osnovy fiziki plasmy”, MIR Ed., Moscow, 1983.

[8] S. Ichimaru, "Statistical Plasma Physics", Addison-Wesley, Redwood, 1992.

[9] D. D. Clayton et al., Astrophys. J., 199 (1975) 194; G. Kaniadakis et al., Physica A, 261 (1998) 359.

[10] M. Coraddu et al., Brazilian Journal of Physics, 29 (1999) 153.

[11] F. Ferro, A. Lavagno and P. Quarati, Physica A 340 n. 1-3 (2004) 477.

[12] F. Ferro, Diploma Thesis, Politecnico di Torino, Torino, 2003.

[13] A. Hoffknecht et al., physics/0003088 v. 1, (2000).

[14] G. Maero, P. Quarati and F. Ferro, Eur. Phys. J. B 50 (2006) 23.

[15] L. Kadanoff et al., "Quantum Statistical Mechanics", Benjamin, NY, 1962.

[16] V. M. Galitskî̌ et al., JETP 51 (1966) 957 and JETP, 24 (1967) 3.

[17] C. Beck, Phys. Rev. Lett. 87 (2001) 180601.

[18] D. R. Present, "Kinetic Theory of Gases", Mc-Graw Hill, NY, 1958. 